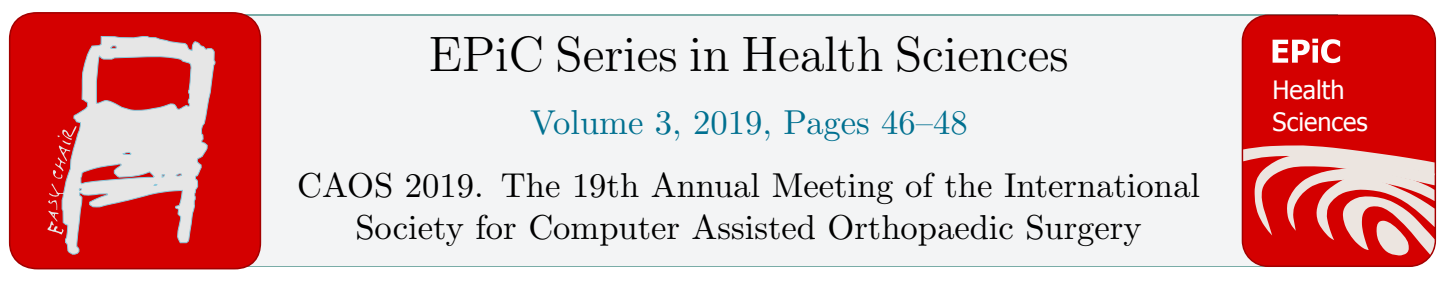

\title{
How Surgeons Perform Total Knee Arthroplasty? A Global Appraisal of Computer- Assisted Surgeries
}

\author{
Charlotte Bolch ${ }^{1 *}$, Yifei Dai ${ }^{*}$, Amaury Jung 2 , Cyril Hamad ${ }^{2}$ \\ ${ }^{1}$ Exactech Inc, Gainesville, FL 32653, USA \\ ${ }^{2}$ Blue Ortho, Gieres, FR \\ charlotte.bolch@exac.com yifei.dai@exac.com
}

\begin{abstract}
This study investigated global variations in the surgical methods used with a contemporary CAOS system in TKA. Individual surgical profiles were identified from a database of technical reports on all TKA cases performed using a CAOS system. The profiles were divided into groups based on geographic regions (US, EU, and APAC), implant types (CR and PS), and application years. Differences were found in the surgeons' preferences of surgical parameters and methods, reflecting the existence of variabilities in how surgeons perform TKA.
\end{abstract}

\section{Introduction}

Computer-assisted orthopaedic surgery (CAOS) offers increased accuracy and precision to total knee arthroplasty (TKA) compared to the conventional techniques [1-3]. Several meta-analyses have assessed the alignment and/or patient outcomes across multiple clinical sites [2,3]. However, a surgeon's choice of surgical method is multifactorial and may be dependent on geographic regions, TKA implant types (CR: cruciate-retaining and PS: posterior-stabilizing), and the potential evolution of surgical philosophy over time. To date, limited information is available on the variations of surgical parameters and methods across the surgeon population.

A comprehensive understanding of how surgeons perform TKA globally may shed a light on improved interpretation of clinical outcomes. Furthermore, the knowledge may offer potential opportunities for the improvement in the design and development of CAOS systems that addresses operative needs from specific patient populations. As the application of CAOS TKA increases [3], technical data has been collected globally on the surgeries. The data contains not only the intraoperative measurements, but also surgical steps and preferences that provides unprecedented opportunity to drive the knowledge forward on the surgical methods applied on TKA surgeries. The purpose of this study was to investigate global variations in the surgical parameters and methods used with a contemporary CAOS system in TKA. 


\section{Materials and Methods}

A retrospective review was carried out on the technical records of more than 10,000 TKA cases performed between December 2010 and August 2018 using a contemporary CAOS system (no patient information was reviewed). The CAOS system allows the set-up of personalized surgical profiles based on surgeon's preferences. These profiles encompass an extensive set of surgical parameters and methods including the surgical steps to be navigated, the sequence of the steps, the definition of the anatomical references, and the parameters associated with the targeted resections. All individual surgical profiles were identified from the database and divided into groups based on (1) geographic regions: United States (US), Europe (EU: Switzerland, France, Italy, United Kingdom, Spain), and Asia (APAC: Australia, India, Japan, South Korea, Singapore), (2) implant types (CR and PS) and (3) application years. A detailed analysis of each group was carried out and compared on the surgical profiles.

\section{Results}

A summary of the choices of resection parameters from a total of 851 individual surgical profiles is presented in Figure 1. A neutral varus/valgus alignment was predominately targeted across all investigated groups for both tibia and femur. A noticeable but very limited trend of targeting a slight residual varus/valgus alignment was observed, which may align with the introduction of kinematic alignment philosophy around 2012 (Fig. 1A,B). The spread of this trend from its origin (US) to other geographic regions over time was observed. The surgeon's choice of tibia slope is predominately $3^{\circ}$ across all geographic regions and TKA implant types, expect that higher percentage of APAC surgeons prefer to use greater slope (greater than $3^{\circ}$ ) for CR TKAs (46.8\%) (Fig. 1C,D). The majority of EU surgeons (CR: $87.0 \%$, PS: 80.4\%) prefer to resect the distal femur in neutral flexion/extension. In contrast, US (CR: $83.3 \%$, PS: $82.2 \%$ ) and APAC (CR: 83.9\%, PS: 69.7\%) surgeons prefer to resect the distal femur with a slight degree of flexion (mostly by $2^{\circ}$ ).

The order of bony resections and soft tissue balancing demonstrated geographic and implant type differences (Fig. 2). Specifically, EU surgeons predominately perform tibial cut first (CR: $85.2 \%$, PS: $67.1 \%$ ) which may be associated with a higher usage of partial or full gap balancing in their TKA cases. EU surgeons also preferred gap balancing more for CR surgeries (70.4\%) than PS surgeries $(55.1 \%)$. Notably different, APAC surgeons tended to do more measured resections (CR: 69.4\%, PS: $70.0 \%$ ) than partial or full gap balancing (CR: $30.6 \%$, PS: $33.0 \%$ ). US surgeons exhibited a relatively more balanced choices between gap balancing and measured resection.

\section{Discussion}

This study demonstrated that geographic, implant type, and historical differences existed in the surgeons' preference of surgical parameters and methods during TKA. These differences may reflect global variations in surgeon training, surgical philosophy, or the specific characteristics of the patient population, which warrants further investigation. It remains to be investigated if the variations lead to clinically different results.

Although alignment and patient outcomes have been the focus of the current research on CAOS TKA [1-3], variations in surgical profiles should not be overlooked, as published smaller scoped studies have shown geographic-dependency in patient population characteristics, application trend, and surgeon treatment choice in TKA [4-6]. The strength of this study was that it covered all the available surgical profiles spanning the application history of a specific CAOS system. As such, variability due to the operational differences of multiple systems was avoided. The findings may inform further 
improvement of design and development of instrument systems (hardware and/or software) that suit global needs, both for conventional and CAOS TKAs.

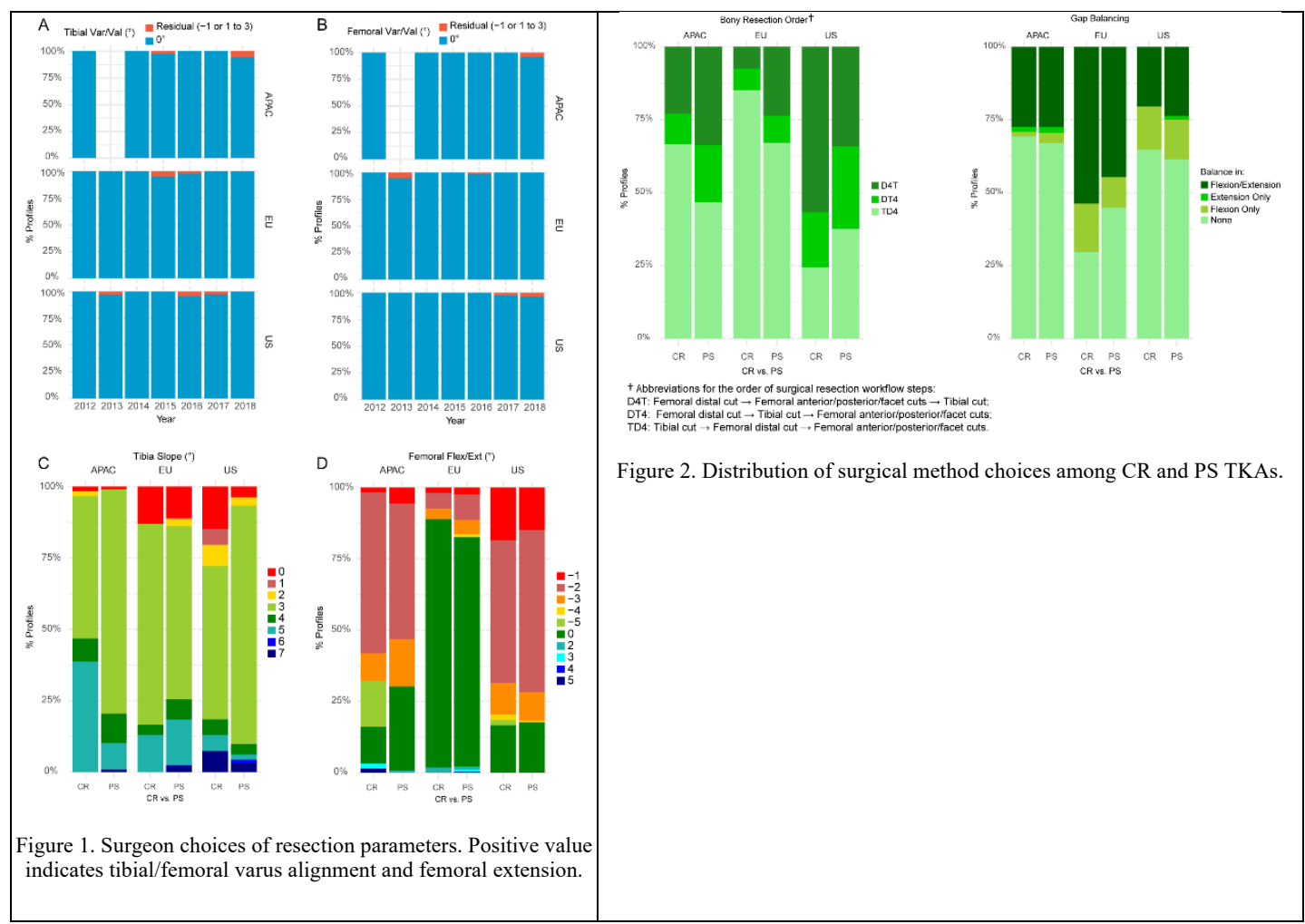

\section{References}

[1] Sparmann M, et al. J Bone Joint Surg Br, 85(6):830-5, 2003.

[2] Brin YS, et al. Int Orthop, 35(3):331-9, 2011.

[3] Bala A, et al., J Knee Surg, 2016; 29(05): 430-5

[4] Robertsson O, et al. Bull Hosp Jt Dis, 58(3):133-8, 1999.

[5] van Walraven CV, et al. Cmaj, 155(6):697-706, 1996.

[6] Wright JG, et al. Med Care, 37(9):946-56, 1999. 